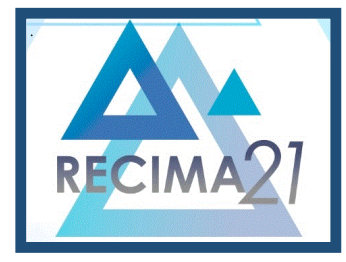

\author{
RECIMA21 - REVISTA CIENTÍFICA MULTIDISCIPLINAR \\ ISSN 2675-6218
}

\title{
ATUAÇÃO DO ENFERMEIRO FRENTE A PREVENÇÃO DO CÂNCER DE PÊNIS NA ATENÇÃO PRIMÁRIA DE SAÚDE: UMA REVISÃO DA LITERATURA
}

\author{
NURSES' PERFORMANCE IN RELATION TO THE PREVENTION OF PENILE CANCER IN \\ PRIMARY HEALTH CARE: A LITERATURE REVIEW
}

\section{DESEMPEÑO DEL ENFERMERO EN RELACIÓN A LA PREVENCIÓN DEL CÁNCER DE PENE EN LA ATENCIÓN PRIMARIA DE SALUD: UNA REVISIÓN DE LA LITERATURA}

\begin{abstract}
Joelma Zoroastro dos Santos Souza ${ }^{1}$, Marluci Conceição dos Santos Almeida ${ }^{2}$, Wanderson Alves Ribeiro ${ }^{3}$ Lilian Laine da Conceição Dias ${ }^{4}$, Renata Alves Fonseca ${ }^{5}$, Vinícius Santos Braz da Cunha ${ }^{6}$, Greyce Kelly Souza Motta Alcoforado ${ }^{7}$, Elisa Santana Daniel ${ }^{8}$, Érica Souza do Nascimento ${ }^{9}$, Amanda Gomes de Moura Duarte $^{10}$
\end{abstract}

\begin{abstract}
RESUMO
O câncer de pênis é um tipo raro, com alta incidência em homens da terceira idade, porém, podem ser afetados os mais jovens. A doença acomete indivíduo de baixo nível social, com maus hábitos higiênicos, tendo como principal fator de risco a fimose e muitas das vezes estão associadas ao papilomavírus humanos (HPV). O atraso na procura por ajuda médica, o tratamento tardio na maioria dos casos, o câncer de pênis terá que submeter a cirurgia, ocorrendo muitas vezes a penectomia total ou parcial e metástase, alternativa essa que afeta a saúde psíquica, física do homem e de toda família. A maioria já ouviu falar sobre o câncer de pênis e sabem que a higiene íntima com água e sabão, a circuncisão, o uso de preservativo são maneiras mais eficazes de prevenção. Trata-se de uma pesquisa bibliográfica de abordagem qualitativa e caráter descritivo, que objetivou compreender as práticas e saberes do enfermeiro na prevenção ao câncer de pênis na Atenção Primária de Saúde. Os resultados apontam que a educação em saúde é uma das principais ferramentas para a prevenção do câncer de pênis. Conclui-se que, a promoção da saúde é uma estratégia relevante frente ao câncer de mama e que o enfermeiro, quando identifica os grupos vulneráveis, pode estabelecer medidas preventivas e educação em saúde para aqueles que estão em risco da doença.
\end{abstract}

PALAVRAS-CHAVE: Atenção primária da saúde. Enfermagem. Educação em Saúde. Câncer de Pênis. Saúde do Homem

\footnotetext{
${ }^{1}$ Enfermeira. Graduada em Enfermagem pela Universidade Iguaçu (UNIG)

${ }^{2}$ Enfermeira. Graduada em Enfermagem pela Universidade Iguaçu (UNIG)

${ }^{3}$ Enfermeiro. Mestre e Doutorando pela Programa Acadêmico em Ciências do Cuidado em Saúde (PACCS) da Universidade federal Fluminense (UFF); Pós-graduação em Enfermagem em Oncologia pelo IBRA. Docente no curso de Graduação em Enfermagem e Pós-Graduação em Enfermagem em Obstetrícia, Emergência e Terapia Intensiva da Universidade Iguaçu (UNIG)

${ }^{4}$ Enfermeira. Pós-Graduada em Enfermagem em Oncologia pela UCL

5 Enfermeira. Graduada em Enfermagem pela Universidade Iguaçu (UNIG)

${ }^{6}$ Enfermeiro. Graduado em Enfermagem pela Universidade Iguaçu (UNIG)

7 Enfermeira. Graduada em Enfermagem pela Universidade Iguaçu (UNIG)

${ }^{8}$ Enfermeira. Graduada em Enfermagem pela Universidade Iguaçu (UNIG)

${ }^{9}$ Enfermeira. Graduada em Enfermagem pela Universidade Iguaçu (UNIG)

${ }^{10}$ Enfermeira. Graduada em Enfermagem pela Universidade Iguaçu (UNIG)
} 


\title{
RECIMA21 - REVISTA CIENTÍFICA MULTIDISCIPLINAR ISSN 2675-6218
}

\author{
ATUAÇÃO DO ENFERMEIRO FRENTE A PREVENÇÃO DO CÂNCER DE PÊNIS NA \\ ATENÇÃO PRIMÁRIA DE SAÚDE: UMA REVISÃO DA LITERATURA \\ Wanderson Alves Ribeiro, Joelma Zoroastro dos Santos Souza, Marluci Conceição dos Santos Almeida, \\ Lilian Laine da Conceição Dias, Renata Alves Fonseca, Vinícius Santos Braz da Cunha, \\ Érica Souza do Nascimento, Amanda Gomes de Moura Duarte
}

\begin{abstract}
Penile cancer is a rare type, with a high incidence in men of the third age, however, it can be affected in younger people. The disease affects individuals of low social level, with poor hygienic habits, having phimosis as the main risk factor and often associated with human papillomavirus (HPV). The delay in seeking medical help, late treatment in most cases, penile cancer will have to undergo surgery, with total or partial penectomy and metastasis often occurring, an alternative that affects the psychological, physical and human health of men and women and the whole family. Most have heard about penile cancer and know that intimate hygiene with soap and water, circumcision, and condom use are the most effective means of prevention. This is a bibliographic research with a qualitative approach and descriptive character, which aimed to understand nurses' practices and knowledge in the prevention of penis cancer in Primary Health Care. The results indicate that health education is one of the main tools for the prevention of penile cancer. We conclude that health promotion is a relevant strategy in the face of breast cancer and that nurses, when identifying vulnerable groups, can establish preventive measures and health education for those who are at risk of the disease.
\end{abstract}

KEYWORDS: Primary health care. Nursing. Health education. Penile Cancer. Men's Health.

\section{RESUMEN}

El cáncer de pene es un tipo poco común, con una alta incidencia en hombres de la tercera edad, sin embargo, puede verse afectado en personas más jóvenes. La enfermedad afecta a individuos de bajo nivel social, con malos hábitos higiénicos, teniendo la fimosis como principal factor de riesgo y frecuentemente asociada al virus del papiloma humano (VPH). El retraso en la búsqueda de ayuda médica, tratamiento tardío en la mayoría de los casos, el cáncer de pene tendrá que ser intervenido quirúrgicamente, con penectomías totales o parciales y metástasis frecuentes, una alternativa que afecta la salud psicológica, física y humana de hombres y mujeres. La mayoría ha oído hablar del cáncer de pene y sabe que la higiene íntima con agua y jabón, la circuncisión y el uso de condones son los medios de prevención más eficaces. Se trata de una investigación bibliográfica con enfoque cualitativo y carácter descriptivo, que tuvo como objetivo comprender las prácticas y conocimientos del enfermero en la prevención del cáncer de pene en Atención Primaria de Salud. Los resultados indican que la educación en salud es una de las principales herramientas para la prevención del cáncer de pene. cáncer. Se concluye que la promoción de la salud es una estrategia relevante ante el cáncer de mama y que las enfermeras, al identificar a los grupos vulnerables, pueden establecer medidas preventivas y de educación para la salud de quienes se encuentran en riesgo de contraer la enfermedad.

PALABRAS CLAVE: Primeros auxilios. Enfermería. Educación para la salud. Cáncer de pene. Salud de los hombres

\section{INTRODUÇÃO}

O câncer de pênis é uma neoplasia rara, que atinge aproximadamente 1/100.000 homens nos países desenvolvidos. A alta incidência é observada principalmente em homens na terceira idade, independentemente de sua origem étnica. No entanto, indivíduos jovens também podem ser afetados, uma vez que aproximadamente $22 \%$ dos casos são registrados em pacientes com idade inferiores a quarenta anos. A doença acomete indivíduos de baixo nível social, com maus 


\title{
RECIMA21 - REVISTA CIENTÍFICA MULTIDISCIPLINAR ISSN 2675-6218
}

\author{
ATUAÇÃO DO ENFERMEIRO FRENTE A PREVENÇÃO DO CÂNCER DE PÊNIS NA \\ ATENÇÃO PRIMÁRIA DE SAÚDE: UMA REVISÃO DA LITERATURA \\ Wanderson Alves Ribeiro, Joelma Zoroastro dos Santos Souza, Marluci Conceição dos Santos Almeida, \\ Lilian Laine da Conceição Dias, Renata Alves Fonseca, Vinícius Santos Braz da Cunha,

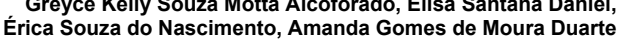

hábitos de higiene e não circuncidados, tendo como principal fator de risco a fimose e muitas vezes estão associadas ao papilomavírus humano (HPV) (Gao et al., 2016).

Em países onde a circuncisão neonatal é um hábito cultural, verifica-se que a incidência do carcinoma de células escamosas do pênis (CCE) é baixa. A higiene adequada e a circuncisão precoce previnem a ocorrência da neoplasia na idade adulta. A presença de fimose é encontrada em aproximadamente $85 \%$ dos pacientes com câncer de pênis, e incluem o carcinoma de origem escamosa (Brasil, 2018).

Globalmente encontram-se focos maiores do câncer de pênis em locais em desenvolvimento ou subdesenvolvidos, como Ásia, América do Sul e África, com aproximadamente 26.000 novos casos por ano (Janini et al., 2014; Gao et al., 2016; Coelho et al., 2018; Olesen et al., 2019).

Estima-se que a incidência seja de 8.3 em cada 100.000 homens brasileiros, sendo uma das nações com maior índice no mundo. No Brasil as regiões mais afetadas são a Norte e Nordeste, sendo regiões com elevada desigualdade social e extrema pobreza. Aliados a tal cenário, destaca-se a falta de higiene íntima, fimose, vírus do papiloma (HPV), uso de tabaco e promiscuidade sexual, como fatores importantes para o desenvolvimento da doença (Coelho \& Miranda, 2018).

Nos casos de avanços da enfermidade, o tratamento comumente utilizado é a remoção cirúrgica do local afetado, podendo evoluir para amputação parcial ou total do órgão. Entretanto, técnicas poupadas têm sido amplamente discutidas nesse universo, como radioterapia, quimioterapia e a cirurgia a laser (Brasil, 2013).

Segundo o Instituto Nacional do Câncer, o câncer de pênis é um tumor raro, com maior incidência em homens a partir dos 50 anos, embora possa atingir também os jovens. Está relacionado às baixas condições socioeconômicas, má higiene íntima, em homens que não se submeteram a circuncisão (remoção do prepúcio, ele que reveste a glande a "cabeça do pênis") (Brasil, 2013).

Diante disso, vale complementar que o estreitamento do prepúcio é um fator de predisposição ao câncer peniano. Estudos científicos também sugerem a associação entre infecção pelo vírus do HPV e o câncer de pênis. Embora se estime que a incidência seja muito menor do que o da próstata, esse câncer exige, muitas vezes, a amputação do pênis. A metástase também costuma ser frequente nesses homens devido os mesmos demorarem a fazerem a procura de tratamento no início. E o aumento de casos de câncer de pênis no Nordeste do país faz sua incidência ser considerada um problema de saúde pública (Brasil, 2013).

No Brasil, o tumor representa $2 \%$ de todos os casos de câncer no homem, sendo, mais frequente nas regiões Norte e Nordeste. Entretanto, nas regiões de maior incidência, o câncer de pênis supera os casos de câncer de próstata e de bexiga (Brasil, 2009). As causas do câncer de 


\title{
RECIMA21 - REVISTA CIENTÍFICA MULTIDISCIPLINAR ISSN 2675-6218
}

\author{
ATUAÇÃO DO ENFERMEIRO FRENTE A PREVENÇÃO DO CÂNCER DE PÊNIS NA \\ ATENÇÃO PRIMÁRIA DE SAÚDE: UMA REVISÃO DA LITERATURA \\ Wanderson Alves Ribeiro, Joelma Zoroastro dos Santos Souza, Marluci Conceição dos Santos Almeida, \\ Lilian Laine da Conceição Dias, Renata Alves Fonseca, Vinícius Santos Braz da Cunha, \\ Greyce Kelly Souza Motta Alcoforado, Elisa Santana Daniel,
} pênis incluem higiene precária e acúmulo de esmaga sob o prepúcio fimótico, resultando em inflamação crônica (Paula; Souza et al., 2012). Há cerca de mil amputações por ano do órgão, o número de casos no Brasil é alto e subnotificado. Deve ter muito mais, mas não se sabe nada sobre as estatísticas nacionais. São mil amputações por ano no Brasil, números semelhantes só ao de Urgência (Brasil, 2011).

De acordo com a esta estatística, cabe mencionar que, além disso, a população masculina, por buscar tardiamente os serviços de saúde, sofre com condições severas e crônicas. A incipiente procura desses serviços, sobretudo no que concerne aos aspectos preventivos dos agravos, tornou-se um fator preponderante para os diagnósticos tardios de inúmeras doenças, como o câncer de pênis, que acomete um número crescente de homens (Costa et al., 2015).

Dentro desse contexto, a Política Nacional de Atenção Integral a Saúde do Homem (PNAISH), reconhece barreiras que dificultam à procura do homem pelos serviços de saúde, tais como as de ordem institucional, problemas quanto à acessibilidade, além do visível despreparo dos profissionais de saúde para receber esses indivíduos, respeitando suas particularidades, o que contribui para ações que impossibilitam a construção de vínculos entre os homens e esses profissionais (Brasil, 2008; Costa et al., 2015).

Nascimento et al., (2018) referem que os homens de forma geral, evitam o contato com os ambientes de saúde, sejam os ambulatórios médicos ou os corredores das unidades de saúde pública, orgulhando-se da oportuna invulnerabilidade. Contrários à precaução e ao autocuidado, sendo comum que adiem a procura de atendimento, consentindo que os casos de patologias simples se agravem e, consequentemente, venham a tornar mais sérios, levando-os muitas das vezes ao confinamento hospitalar. $\mathrm{Na}$ maioria das vezes, onerando gastos para si e para o Sistema Único de Saúde (SUS), que é obrigado a intervir nas fases mais avançadas das doenças, chegando finalmente ao setor terciário de saúde

Dessa forma, Teixeira et al., (2007) diz que o enfermeiro tem um papel muito importante na equipe multidisciplinar realizando ações que previnem e promovem a saúde, dando atenção e cuidando das famílias a sua volta, oferecendo uma boa assistência em enfermagem. A promoção da saúde requer uma união e aplicação de vários saberes e habilidades dos profissionais, sendo necessária uma atenção maior na educação permanente em saúde. A prevenção tem como estruturar-se por meio de divulgação de informações, assim como, recomendações normativas de mudanças de hábitos (Teixeira et al., 2007).

Como formas de prevenção podem ser destacadas ações simples, como a boa higienização do órgão (sendo este o foco da campanha das inúmeras entidades de saúde), a cirurgia de fimose em casos específicos, utilização de preservativos em relação sexuais e o diagnóstico precoce da patologia (Couto et al., 2014). 


\title{
RECIMA21 - REVISTA CIENTÍFICA MULTIDISCIPLINAR ISSN 2675-6218
}

\author{
ATUAÇÃO DO ENFERMEIRO FRENTE A PREVENÇÃO DO CÂNCER DE PÊNIS NA \\ ATENÇÃO PRIMÁRIA DE SAÚDE: UMA REVISÃO DA LITERATURA \\ Wanderson Alves Ribeiro, Joelma Zoroastro dos Santos Souza, Marluci Conceição dos Santos Almeida, \\ Lilian Laine da Conceição Dias, Renata Alves Fonseca, Vinícius Santos Braz da Cunha, \\ Greyce Kelly Souza Motta Alcoforado, Elisa Santana Daniel,
Érica Souza do Nascimento, Amanda Gomes de Moura Duarte
}

A educação destinada ao diagnóstico precoce do câncer de pênis, dever ser priorizada como um compromisso profissional ao indivíduo, devendo ser enfatizada como uma ação que direciona a orientação e prática do enfermeiro destinado à prevenção e o diagnóstico precoce favorecendo a autonomia do paciente (Santos, 2015).

Nesse sentido, a ação preventiva deste tipo de câncer de pênis é um dos primordiais compromissos que o profissional enfermeiro deve possuir com a qualidade de vida populacional, a educação em saúde executada pelo profissional enfermeiro é uma atividade a ser desenvolvida nos serviços de saúde, que orienta e proporciona a redução de sequelas e mortes (Janini et al., 2015).

Sendo de grande prevalência a intensificação de campanha de prevenção, concernentes com a associação de hábitos de higiene precária e a severidade da incidência do câncer do pênis. A utilização preservativa favorece na diminuição do desenvolvimento de infecção sexualmente transmissível (IST), tendo como exemplo o HPV (Barbosa et al., 2015).

O enfermeiro enquanto profissional direcionado de modo essencial ao cuidar do desenvolvimento de ações de promoção, proteção e recuperação da saúde, seja de caráter individual ou coletivo, contribui amplamente na adesão dos indivíduos ao autocuidado, exercendo atribuição de grande ênfase, sendo incrementadas nesse objetivo do controle de sequelas (Coelho \& Miranda, 2015).

Como educador, o enfermeiro deve promover o desenvolvimento de estratégias de prevenção que favoreçam a necessidade dos indivíduos. A educação em saúde é uma importante estratégia de prevenção, ou seja, é instruir o indivíduo acerca de questões que precisam ser inseridas dentro do contexto vivenciado por eles, incluir a educação como meio de prevenção, a detecção precoce e assim, auxiliando para um diagnóstico positivo e consequentemente a redução de sequelas físicas, psicológicas e sociais (Sousa et al., 2015; Salci et al., 2016).

Dentre os profissionais da área da saúde, é o enfermeiro que permanece maior parte do tempo com os pacientes no processo de saúde doença, tornando-se seu papel primordial para o sucesso do tratamento (Camargos; Belhiane \& Matos, 2014).

O aspecto preventivo assume três vertentes, classificados em primária, secundária, terciária. A primária faz referência ao uso adequado de preservativos, às campanhas informativas, especialmente sobre o uso de tabaco e higiene pessoal, e à vacinação contra HPV.

A vertente secundária abrange o diagnóstico precoce e o tratamento em si, avaliação do caso e utilização da melhor técnica, sendo citado a penectomia, o uso de biomarcadores e, em casos específicos, a segmentação do genoma do HPV (Scheed et al., 2018). Pode-se destacar, também, que a melhor da sobrevivência em homens com câncer de pênis inclui o fato de serem casados. Supõe-se que homens casados, ou em um relacionamento, tenham suas lesões penianas identificadas anteriormente e presentes para atendimento médico precocemente (Pietro, 


\title{
RECIMA21 - REVISTA CIENTÍFICA MULTIDISCIPLINAR ISSN 2675-6218
}

\author{
ATUAÇÃO DO ENFERMEIRO FRENTE A PREVENÇÃO DO CÂNCER DE PÊNIS NA \\ ATENÇÃO PRIMÁRIA DE SAÚDE: UMA REVISÃO DA LITERATURA \\ Wanderson Alves Ribeiro, Joelma Zoroastro dos Santos Souza, Marluci Conceição dos Santos Almeida, \\ Lilian Laine da Conceição Dias, Renata Alves Fonseca, Vinícius Santos Braz da Cunha, \\ Greyce Kelly Souza Motta Alcoforado, Elisa Santana Daniel,
} 2017). Existe ainda a discussão sobre a circuncisão como forma de prevenção primaria, porém, os estudos são inconclusivos e em sua maioria afirma ser uma opção extremamente invasiva (Pietro, 2017).

Além disso, o atraso na procura por ajuda médica desde o início dos primeiros sintomas é motivo de grande preocupação dos profissionais da saúde. Considerando o diagnóstico precoce como segunda melhor prevenção, além da assepsia diária do órgão, tal cenário é real graças a três principais motivos: os pacientes acreditam que os sintomas irão sumir sozinhos (28\%) sentem -se desconfortáveis em procura ajuda médica (23\%) e creem que o problema não é grave (19\%) (Gao et al., 2016).

Partindo daí, surgiu o interesse de investigar sobre a temática do câncer de pênis e também sobre o conhecimento de cuidados adotados com estudos em equipe de enfermagem, ao paciente com câncer de pênis. Como base nisso, este estudo tem como objetivo geral: Compreender as práticas e saberes do enfermeiro na prevenção ao câncer de pênis na Atenção Primária de Saúde e como objetivos específicos: identificar as principais estratégias de educação em saúde, utilizadas pelo enfermeiro, na prevenção ao câncer de pênis na Atenção Primária de Saúde e Descrever as práticas e saberes do enfermeiro na prevenção ao câncer de pênis na Atenção Primária de Saúde.

Frente a isso, emergiu a seguinte questão norteadora: quais são as práticas e saberes do enfermeiro na prevenção ao câncer de pênis na Atenção Primária de Saúde?

\section{METODOLOGIA}

Todas as ciências caracterizam-se pela utilização de métodos científicos; em contrapartida, nem todos os ramos de estudo que empregam estes métodos são ciências. Dessas afirmações podemos concluir que a utilização de métodos científicos não é da alçada exclusiva da ciência, mas não há ciência sem o emprego de métodos científicos (Lakatos \& Marcone, 2003).

Perante a certificação, pode-se deduzir que a aplicação de métodos científicos não é competência especifica da ciência, com tudo não existe ciência sem o uso de métodos científicos. Como tal característica, o método é a agregação de atividades sistemáticas e lógicas que, permite com total segurança e economia, atingir o objetivo, com estudos válidos e verdadeiros, elaborando roteiros a serem seguidos, encontrando erros e contribuindo com soluções dos cientistas (Lakatos \& Marcone, 2003).

$\mathrm{Na}$ atualidade têm-se uma farta e complexa quantidade de dados na área da saúde, fazendo assim, com que haja necessidade de desenvolvimento de artigos e pesquisas, com embasamento cientifico, para possibilitar melhor delimitação metodológica, esclarecendo diversos estudos. Mediante a necessidade, utilizamos a revisão bibliográfica como uma forma de 


\title{
RECIMA21 - REVISTA CIENTÍFICA MULTIDISCIPLINAR ISSN 2675-6218
}

\author{
ATUAÇÃO DO ENFERMEIRO FRENTE A PREVENÇÃO DO CÂNCER DE PÊNIS NA \\ ATENÇÃO PRIMÁRIA DE SAÚDE: UMA REVISÃO DA LITERATURA \\ Wanderson Alves Ribeiro, Joelma Zoroastro dos Santos Souza, Marluci Conceição dos Santos Almeida, \\ Lilian Laine da Conceição Dias, Renata Alves Fonseca, Vinícius Santos Braz da Cunha, \\ Greyce Kelly Souza Motta Alcoforado, Elisa Santana Daniel,
}

metodologia que possibilita um apanhado de conhecimentos e aplica-se em resultados de estudos concisos não práticos do profissional (Minayo, 2013).

Trata-se de um estudo de revisão bibliográfica, de caráter descritivo e de abordagem qualitativa. A pesquisa científica é a atuação básica das ciências na sua indagação e construção da realidade, tornando-a uma atividade expressiva (Minayo, 2013).

Abordagem qualitativa é aquela que não trabalha com informações numéricas, mas sim, que trabalha com conceitos, ideologias, processos de comunicação humana, entre outros. $E$ apresenta facilidade de definir hipótese ou problema, de explorar a interação de certas variáveis, de compreender e classificar processos dinâmicos experimentados por grupos sociais, de apresentar mudanças, elaboração ou formação de posição de determinados grupos, e de permitir, em grau de profundidade, a interpretação dos comportamentos ou atitudes dos indivíduos (Gil, 2008).

Foram realizadas buscas na base de dados da Biblioteca Virtual em Saúde (BVS) - Bireme, entre junho e novembro, 2019, nas bases de dados: Literatura Lática Americana e do Caribe em Ciência da Saúde (LILACS), Bases de Dados da Enfermagem (BDENF), Biblioteca Eletrônica Cientifica Online (SciELO).

Para a busca das referências foram utilizados os descritores "cuidados paliativos", "enfermeiros", "oncologia", "processos de enfermagem", "equipe de enfermagem", advindos do sistema de Descritores em ciências da saúde (DeCS), utilizando o marcador "AND". Para regaste dos artigos, consideramos como critérios para inclusão artigos publicados no período compreendido entre os anos 2015 e 2019 com textos completos em língua portuguesa. E os critérios de exclusão foram os artigos repetidos, publicações com textos não disponíveis, fora da língua vernácula e estudos com mais de dez anos de publicação.

Inicialmente foram pesquisados os descritores individualmente, sendo encontrados artigos científicos conforme Quadro 1, a seguir:

Quadro 01- Descritores Isolados.

\begin{tabular}{|l|l|l|l|l|}
\hline DESCRITORES & BDENF & LILACS & MEDLINE & $\begin{array}{l}\text { TOTAL } \\
\text { ARTIGOS DE }\end{array}$ \\
\hline Enfermagem & 31450 & 40804 & 529636 & 601890 \\
\hline Saúde do homem & 19356 & 98808 & 3317819 & 3435983 \\
\hline Câncer de pênis & 08 & 292 & 7207 & 7507 \\
\hline
\end{tabular}

Fonte: Construção dos Autores, 2020. 


\section{RECIMA21 - REVISTA CIENTÍFICA MULTIDISCIPLINAR ISSN 2675-6218}

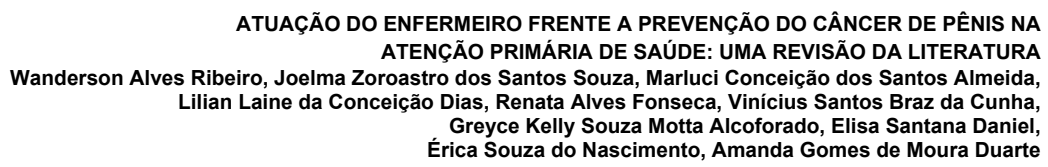

Diante do extenso número de publicações encontradas, realizou-se um refinamento na busca. Os descritores foram pesquisados de forma associada em dupla, utilizando o termo "and", conforme Quadro 2:

Quadro 2 - Distribuição quantitativa das produções científicas encontradas nas bases de dados com descritores associados em dupla.

\begin{tabular}{|l|l|l|l|l|}
\hline \multicolumn{5}{|c|}{ BANCO DE DADOS } \\
\hline DESCRITORES & LILACS & BDENF & MEDLINE & TOTAL \\
\hline Enfermagem and Saúde do homem & 13358 & 12479 & 153888 & 179725 \\
\hline Enfermagem and Câncer de pênis & 06 & 05 & 27 & 38 \\
\hline $\begin{array}{l}\text { Saúde do homem and Câncer de } \\
\text { pênis }\end{array}$ & 41 & 06 & 1066 & 1113 \\
\hline
\end{tabular}

Fonte: Construção dos Autores, 2020.

Considerando ainda ser extensa a quantidade de produções científicas, optou-se pela busca com os descritores associados em trio. Os resultados dessa busca se encontram descritos no Quadro 3.

Quadro 3 - Distribuição quantitativa das produções científicas encontradas nas bases de dados com os descritores associados em trio.

\begin{tabular}{|c|l|l|l|l|}
\hline \multicolumn{5}{|c|}{ BANCO DE DADOS } \\
\hline DESCRITORES & LILACS & BDENF & MEDLINE & TOTAL \\
\hline $\begin{array}{c}\text { Enfermagem and Saúde do homem } \\
\text { and Câncer de Pênis }\end{array}$ & 03 & 04 & 08 & 15 \\
\hline
\end{tabular}

Fonte: Construção dos Autores, 2020.

Finalizado esse percurso de busca, realizou-se a leitura dos resumos e os que apresentavam relevância para subsidiar a discussão do tema foram selecionados e lidos na íntegra.

A partir dessa leitura preliminar, foram selecionados 9 artigos que mantinham coerências com os descritores acima apresentados e com os objetivos do estudo. A partir dessa análise, foi extraída a bibliografia potencial, explicitada no Quadro 4 a seguir.

Quadro 04: Levantamento estrutural dos artigos selecionados nas bases de dados da temática

\begin{tabular}{|l|l|l|l|l|l|}
\hline Título & Autores & Objetivo & Revista & Ano & $\begin{array}{l}\text { Principais } \\
\text { conclusões }\end{array}$ \\
\hline $\begin{array}{l}\text { Saberes e } \\
\text { pratica } \\
\text { enfermeiro do }\end{array}$ & $\begin{array}{l}\text { Costa et } \\
\text { al., }\end{array}$ & $\begin{array}{l}\text { Descrever e analisar } \\
\text { o conhecimento e a } \\
\text { pratica do enfermeiro }\end{array}$ & $\begin{array}{l}\text { Rev. } \\
\text { pequi. } \\
\text { Fundam }\end{array}$ & 2015 & $\begin{array}{l}\text { A estratégia saúde da } \\
\text { família configura um } \\
\text { cenário de }\end{array}$ \\
\hline
\end{tabular}




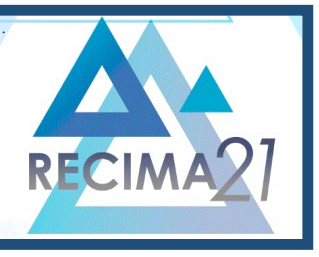

\section{RECIMA21 - REVISTA CIENTÍFICA MULTIDISCIPLINAR ISSN 2675-6218}

ATUAÇÃO DO ENFERMEIRO FRENTE A PREVENÇÃO DO CÂNCER DE PÊNIS NA ATENÇÃO PRIMÁRIA DE SAÚDE: UMA REVISÃO DA LITERATURA Wanderson Alves Ribeiro, Joelma Zoroastro dos Santos Souza, Marluci Conceição dos Santos Almeida, Lilian Laine da Conceição Dias, Renata Alves Fonseca, Vinícius Santos Braz da Cunha, Greyce Kelly Souza Motta Alcoforado, Elisa Santana Daniel, Érica Souza do Nascimento, Amanda Gomes de Moura Duarte

\begin{tabular}{|c|c|c|c|c|c|}
\hline $\begin{array}{l}\text { acerca do } \\
\text { câncer de pênis }\end{array}$ & & $\begin{array}{l}\text { da estratégia saúde } \\
\text { da família sobre o } \\
\text { câncer de pênis. }\end{array}$ & (online) & & $\begin{array}{l}\text { conhecimento acerca } \\
\text { do câncer de pênis } \\
\text { repleto de lacunas, o } \\
\text { que exige reflexões } \\
\text { que possam contribuir } \\
\text { para construção de } \\
\text { uma atenção integra e } \\
\text { integra a saúde do } \\
\text { homem. }\end{array}$ \\
\hline $\begin{array}{l}\text { Diagnostico de } \\
\text { enfermagem em } \\
\text { pacientes } \\
\text { submetidos a } \\
\text { tratamento } \\
\text { cirúrgico para } \\
\text { câncer de pênis }\end{array}$ & $\begin{array}{l}\text { Amorim et } \\
\text { al., }\end{array}$ & $\begin{array}{l}\text { Construir diagnostico } \\
\text { de enfermagem para } \\
\text { pacientes } \\
\text { submetidos a } \\
\text { tratamentos cirúrgico } \\
\text { para câncer de } \\
\text { pênis. }\end{array}$ & $\begin{array}{l}\text { Revista } \\
\text { de } \\
\text { pesquis } \\
\text { a } \\
\text { cuidado } \\
\text { é } \\
\text { Fundam } \\
\text { ental } \\
\text { Online. }\end{array}$ & 2015 & $\begin{array}{l}\text { A enfermagem } \\
\text { reconhece e avalia as } \\
\text { necessidades de } \\
\text { pacientes submetidos } \\
\text { a tratamento cirúrgico } \\
\text { de câncer de pênis nas } \\
\text { dimensões física e } \\
\text { comportamentais. }\end{array}$ \\
\hline $\begin{array}{lr}\text { Estratégias } & \text { do } \\
\text { enfermeiro } & \text { na } \\
\text { prevenção } & \text { do } \\
\text { câncer de pênis }\end{array}$ & $\begin{array}{l}\text { Ramos et } \\
\text { al., }\end{array}$ & $\begin{array}{lr}\text { Identificar } & \text { as } \\
\text { possíveis estratégia } \\
\text { empregadas pelo } \\
\text { enfermeiro r na } \\
\text { prevenção do câncer } \\
\text { de pênis. }\end{array}$ & $\begin{array}{l}\text { Revista } \\
\text { Uniabeu } \\
\text { V.11 }\end{array}$ & 2018 & 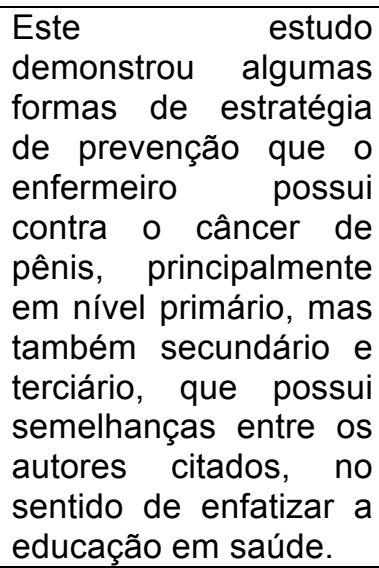 \\
\hline $\begin{array}{l}\text { Câncer de pênis } \\
\text { resultado e } \\
\text { importância de } \\
\text { uma campanha } \\
\text { de prevenção }\end{array}$ & $\begin{array}{l}\text { Correia et } \\
\text { al., }\end{array}$ & $\begin{array}{l}\text { Relatar os resultados } \\
\text { de uma campanha } \\
\text { de combate ao } \\
\text { câncer de pênis, } \\
\text { avaliando o perfil } \\
\text { clinico dos pacientes } \\
\text { e a importância de } \\
\text { campanhas como } \\
\text { estratégia r de } \\
\text { prevenção. }\end{array}$ & $\begin{array}{l}\text { Revist. } \\
\text { Port., } \\
\text { Saúde e } \\
\text { Socieda } \\
\text { de }\end{array}$ & 2018 & $\begin{array}{l}\text { Devido a carência de } \\
\text { informações sobre o } \\
\text { câncer de pênis atinge } \\
\text { a população masculina } \\
\text { de forma dramática. }\end{array}$ \\
\hline $\begin{array}{l}\text { Avaliação do } \\
\text { conhecimento } \\
\text { dos homens } \\
\text { sobre o câncer } \\
\text { de pênis }\end{array}$ & $\begin{array}{l}\text { Chaves } \\
\text { et al., }\end{array}$ & $\begin{array}{lrr}\text { Com } & \text { base } & \text { no } \\
\text { exposto, o objetivo } \\
\text { desse estudo foi } \\
\text { avaliar r o } \\
\text { conhecimento dos } \\
\text { homens com câncer } \\
\text { de pênis. }\end{array}$ & $\begin{array}{l}\text { Revista } \\
\text { Augustu } \\
\text { s }\end{array}$ & 2019 & $\begin{array}{l}\text { Desse segmento } \\
\text { populacional, } \\
\text { principalmente nas } \\
\text { regiões em que a } \\
\text { incidência se constitui } \\
\text { importante problema } \\
\text { de saúde pública }\end{array}$ \\
\hline $\begin{array}{l}\text { Conhecimento } \\
\text { de homens } \\
\text { universitário }\end{array}$ & $\begin{array}{l}\text { Catunda et } \\
\text { al., }\end{array}$ & $\begin{array}{l}\text { Descrever } \\
\text { conhecimento do } \\
\text { homem universitário }\end{array}$ & $\begin{array}{l}\text { Revista } \\
\text { Augustu } \\
\text { s }\end{array}$ & 2019 & $\begin{array}{l}\text { Observa -se } \\
\text { necessidade } \\
\text { estabelecimento }\end{array}$ \\
\hline
\end{tabular}


RECIMA21 - REVISTA CIENTÍFICA MULTIDISCIPLINAR ISSN 2675-6218

ATUAÇÃO DO ENFERMEIRO FRENTE A PREVENÇÃO DO CÂNCER DE PÊNIS NA ATENÇÃO PRIMÁRIA DE SAÚDE: UMA REVISÃO DA LITERATURA Wanderson Alves Ribeiro, Joelma Zoroastro dos Santos Souza, Marluci Conceição dos Santos Almeida, Lilian Laine da Conceição Dias, Renata Alves Fonseca, Vinícius Santos Braz da Cunha, Greyce Kelly Souza Motta Alcoforado, Elisa Santana Daniel, Érica Souza do Nascimento, Amanda Gomes de Moura Duarte

\begin{tabular}{|c|c|c|c|c|c|}
\hline $\begin{array}{l}\text { sobre o câncer } \\
\text { de pênis }\end{array}$ & & $\begin{array}{l}\text { sobre o câncer de } \\
\text { pênis e pratica } \\
\text { preventivas. }\end{array}$ & & & $\begin{array}{l}\text { medidas educativas } \\
\text { afim de esclarecer } \\
\text { essa população sobre } \\
\text { o câncer de pênis } \\
\text { como medidas de } \\
\text { estabelecimento de } \\
\text { cultura de cuidados }\end{array}$ \\
\hline \begin{tabular}{l}
\multicolumn{2}{l}{ Determinantes } \\
sociais $\quad$ de \\
pacientes com \\
neoplasia \\
peniana
\end{tabular} & $\begin{array}{l}\text { Conceição } \\
\text { et al., }\end{array}$ & $\begin{array}{l}\text { Descrever } \\
\text { determinantes } \\
\text { sociais dos pacientes } \\
\text { diagnosticados com } \\
\text { câncer de pênis } \\
\text { atendido em um } \\
\text { hospital universitário }\end{array}$ & $\begin{array}{l}\text { Rev. } \\
\text { Enferm. } \\
\text { UFPE } \\
\text { online }\end{array}$ & 2019 & $\begin{array}{l}\text { Concluir-se que os } \\
\text { determinantes sociais } \\
\text { podem servir de base } \\
\text { para que o enfermeiro } \\
\text { oncológico identifique } \\
\text { grupos vulneráveis e, } \\
\text { assim, estabelecer } \\
\text { medidas preventivas e } \\
\text { educação em saúde } \\
\text { para aqueles que } \\
\text { estão em risco da } \\
\text { doença. }\end{array}$ \\
\hline $\begin{array}{l}\text { Saúde do } \\
\text { homem } \\
\text { conhecimento } \\
\text { de homens } \\
\text { caminhoneiros } \\
\text { sobre o câncer } \\
\text { de pênis }\end{array}$ & $\begin{array}{l}\text { Passos et } \\
\text { al., }\end{array}$ & $\begin{array}{l}\text { Tem como objetivo } \\
\text { analisar } r \text { do } \\
\text { conhecimento } \\
\text { homem } \\
\text { caminhoneiro sobre } \\
\text { o câncer de pênis. }\end{array}$ & $\begin{array}{l}\text { Rev. } \\
\text { cientific } \\
\text { a da } \\
\text { faculdad } \\
\text { e de } \\
\text { educaçã } \\
\text { o e meio } \\
\text { ambient } \\
\text { e. }\end{array}$ & 2019 & $\begin{array}{lr}\text { Ao analisar } & \text { o } \\
\text { conhecimento dos } \\
\text { caminhoneiros sobre o } \\
\text { câncer de pênis, } \\
\text { verificou-se que um } \\
\text { quarto } & \text { dos } \\
\text { entrevistados não } \\
\text { sabiam da existência } \\
\text { da patologia e muitos } \\
\text { desconhecem os } \\
\text { fatores que mais } \\
\text { contribuem para o } \\
\text { surgimento da doença. }\end{array}$ \\
\hline $\begin{array}{l}\text { Diagnostico de } \\
\text { enfermagem no } \\
\text { pós-operatório } \\
\text { de penectomia } \\
\text { por câncer de } \\
\text { pênis. }\end{array}$ & $\begin{array}{l}\text { Soares et } \\
\text { al., }\end{array}$ & $\begin{array}{lr}\text { Analisar } & \text { as } \\
\text { evidencias cientifica } \\
\text { produzida no pós- } \\
\text { operatório do câncer } \\
\text { de pênis }\end{array}$ & $\begin{array}{l}\text { Rev. } \\
\text { Uningá. } \\
\text { Maringa } \\
\text {, v.56 }\end{array}$ & 2019 & $\begin{array}{l}\text { Considerando } \\
\text { importância desse tipo } \\
\text { de neoplasia, novos } \\
\text { estudos poderão ser } \\
\text { realizados a fim de } \\
\text { ampliar } \\
\text { conhecimento do pós- } \\
\text { operatório. }\end{array}$ \\
\hline
\end{tabular}

Fonte: Construção dos Autores, 2020.

Uma vez criadas as categorias de análise, partiu-se para a fase final de inferências dos dados obtidos, mediante o respaldo obtido através da articulação entre o conteúdo verificado nas produções científicas e a atitude crítico-reflexiva das pesquisadoras, através da qual construíramse duas categorias: Principais estratégias de educação em saúde, utilizadas pelo enfermeiro, na prevenção ao câncer de pênis na Atenção Primária de Saúde e Práticas e saberes do enfermeiro na prevenção ao câncer de pênis na Atenção Primária de Saúde. 


\section{RECIMA21 - REVISTA CIENTÍFICA MULTIDISCIPLINAR ISSN 2675-6218}

ATUAÇÃO DO ENFERMEIRO FRENTE A PREVENÇÃO DO CÂNCER DE PÊNIS NA ATENÇÃO PRIMÁRIA DE SAÚDE: UMA REVISÃO DA LITERATURA Wanderson Alves Ribeiro, Joelma Zoroastro dos Santos Souza, Marluci Conceição dos Santos Almeida, Lilian Laine da Conceição Dias, Renata Alves Fonseca, Vinícius Santos Braz da Cunha, Greyce Kelly Souza Motta Alcoforado, Elisa Santana Daniel, Érica Souza do Nascimento, Amanda Gomes de Moura Duarte

\section{RESULTADOS E DISCUSSÃO}

Posterior à leitura reflexiva emergiram duas categorias: Principais estratégias de educação em saúde, utilizados pelo enfermeiro, na prevenção ao câncer de pênis na Atenção Primaria de Saúde; Práticas e saberes do enfermeiro na prevenção ao câncer de pênis na Atenção Primária de Saúde.

\section{Categoria 1 - Principais estratégias de educação em saúde, utilizadas pelo enfermeiro, na prevenção ao câncer de pênis na Atenção Primária de Saúde.}

A educação em saúde articulada com a Política Nacional de Promoção da Saúde (PNPS) é uma ferramenta para combater problemas na sociedade, pois tal Política tem maneiras especificas de agir. Os propósitos e conceitos são adaptados de acordo com o ambiente e comunidade, preservando o seu saber e buscando melhorias na sua qualidade de vida. O modelo atual de educação em saúde é caracterizado pelo diálogo bidirecional, ou seja, entre duas partes envolvidas diretamente no processo de educação (Santos, 2018).

Nesse sentido, a estratégia de prevenção do Câncer de Pênis é direcionada ao paciente e à equipe de enfermagem. Relacionado ao paciente, diversos autores evidenciaram que: a prática de circuncisão na infância, a melhora dos hábitos de higiene íntima diariamente, com água e sabão (em especial na glande após a ejaculação), empoderar os meninos desde cedo a fazer uma higiene de seu pênis corretamente e até mesmo realizar o autoexame mensalmente, são formas direcionadas ao paciente, proporcionando assim uma prevenção eficaz contra neoplasia (Santos, 2018).

Considerando que não há um protocolo de ações da Enfermagem para cuidado e a prevenção do Câncer Pênis, cabe ao enfermeiro conhecer sua população e a partir deste momento, associar seu conhecimento à sua pratica laboral. Portanto, servir de base os aspectos sociais para vulneráveis, e assim, estabelecer medidas preventivas do Câncer Pênis, auxiliando nas campanhas para prevenção da doença (Conceição, 2019).

Pela mesma razão, medidas preventivas na infância, investimento em orientações quanto aos hábitos de higiene adequados, o uso de preservativo durante as relações sexuais, a prática do autoexame são condutas essenciais para o diagnóstico precoce, controle e erradicação desse tipo de neoplasia, porém há resistência por parte dos homens em adotar medidas preventivas, como por exemplo, a baixa incidência do uso de preservativos durante as práticas sexuais. Mesmo tendo conhecimento sobre a importância do uso do preservativo, os homens possuem outros valores impeditivos ao uso como, por exemplo: intimidade, confiança, insegurança e comunicação entre parceiras sexuais (Siqueira; Mantovani \& Kletemberg, 2019). 


\title{
RECIMA21 - REVISTA CIENTÍFICA MULTIDISCIPLINAR ISSN 2675-6218
}

\author{
ATUAÇÃO DO ENFERMEIRO FRENTE A PREVENÇÃO DO CÂNCER DE PÊNIS NA \\ ATENÇÃO PRIMÁRIA DE SAÚDE: UMA REVISÃO DA LITERATURA \\ Wanderson Alves Ribeiro, Joelma Zoroastro dos Santos Souza, Marluci Conceição dos Santos Almeida, \\ Lilian Laine da Conceição Dias, Renata Alves Fonseca, Vinícius Santos Braz da Cunha, \\ Greyce Kelly Souza Motta Alcoforado, Elisa Santana Daniel,
}

Evidentemente, os fatores de risco para desenvolvimento do carcinoma peniano são múltiplos e os principais descritos incluem: a higiene insatisfatória, a presença da fimose, infecção pelo papiloma vírus humano (HPV), entre outras. Todavia, convém mencionar que a neoplasia permanece com etiologia ainda não totalmente clarificada, a ação educativa para a prevenção desse tipo de neoplasia, uma das que constituem as ações básicas de saúde, deve ser compreendida como compromisso profissional com a qualidade de vida da população como um compromisso de qualidade no atendimento, reiterando a autonomia do paciente no que diz respeito às práticas de autoridade (Guimarães et al., 2017).

Eventualmente a prevenção, nesse panorama, deve ser o caminho norteador, como forma de evitar que os homens venham a vivenciar essa experiência com potencial significativo para destruir anseios, desejos, vontades e alterar todo um curso de vida. Para tanto, vislumbrar-se a Estratégia Saúde da Família (ESF) e a prática do enfermeiro nesse cenário, como caminho promissor para o alcance de resultados positivos na prevenção do câncer de pênis. Diante disso, é importante intensificar campanhas de prevenção disseminando o conhecimento, pois a relação entre o câncer e os maus hábitos de higiene assim como a fimose e a infecção pelo papiloma vírus humano, tem um efeito desgastante para o homem. Essas campanhas de prevenção proporcionam ao homem diagnosticar esses cânceres no estágio inicial, podendo assim ter a possibilidade de cura ou aumento da sobrevida (Costa et al., 2015).

Nessa perspectiva o enfermeiro tem um papel muito importante na equipe, realizando ações que previnem e promovam a saúde, dando atenção e cuidando das famílias a sua volta, oferecendo uma boa assistência em enfermagem. A promoção da saúde requer agregação e aplicação de vários saberes e habilidades do profissional, sendo necessária uma atenção maior na educação permanente em saúde. E a prevenção estrutura-se por meio da divulgação de informações assim como recomendações normativas de mudanças de hábitos (Costa et al., 2015).

Por certo, na experiência analisada, surge uma observação para reforçar o assunto de que a prevenção e a detecção precoce, estratégias básicas para o controle do Câncer Pênis, tem como requisito essencial um conjunto de atividades educativas constantes, persistentes e dinâmicas para os homens, segundo seu padrão de valores, crenças, questões culturais, entre outras variáveis. Considera-se que atividades educativas de promoção da saúde devam priorizar a necessidade de mudança de comportamento, tanto por parte dos homens, quanto dos serviços de saúde, priorizando a sensibilização para o autocuidado masculino (Siqueira et al., 2019).

\section{Categoria 2 - Práticas e saberes do enfermeiro na prevenção ao câncer de pênis na Atenção} Primária de Saúde. 
RECIMA21 - REVISTA CIENTÍFICA MULTIDISCIPLINAR ISSN 2675-6218

ATUAÇÃO DO ENFERMEIRO FRENTE A PREVENÇÃO DO CÂNCER DE PÊNIS NA ATENÇÃO PRIMÁRIA DE SAÚDE: UMA REVISÃO DA LITERATURA Wanderson Alves Ribeiro, Joelma Zoroastro dos Santos Souza, Marluci Conceição dos Santos Almeida, Lilian Laine da Conceição Dias, Renata Alves Fonseca, Vinícius Santos Braz da Cunha, Greyce Kelly Souza Motta Alcoforado, Elisa Santana Daniel,

O enfermeiro apresenta papel relevante no processo de promoção, prevenção e no autocuidado. A necessidade de adotar uma escuta qualificada na atenção básica é algo premente, ajudando aos pacientes a obter conhecimento sobre o câncer de pênis, instrumentalizando os homens a prevenir e reconhecer a doença, assim como enfrentarem ou se adaptarem às limitações ocorridas pelo câncer de pênis. Ainda com forma de explicar um conhecimento pouco embasado na teoria sobre o câncer de pênis (Costa et al., 2015).

Demonstra-se, assim, que é um desafio estar com câncer, mas a maior dificuldade está na busca de ajuda e na manutenção do papel masculino. Guia-se, dessa forma, o processo de adoecer pela experiência que cada homem tem com a doença. Entende-se que o enfermeiro é um dos profissionais da saúde que, durante suas práxis, presta assistência à saúde dos homens desde a prevenção, o tratamento, até a sobrevivência, por meio do cuidado e da educação (Conceição et al., 2019).

A enfermidade tão pouco conhecida pelos homens, atingindo principalmente a população de países subdesenvolvidos, ligada à má higiene genital e à infecção pelo papiloma vírus humano (HPV), é conhecida como Câncer de pênis. Diversos autores descrevem que a falta de ou a precária higienização da glande e prepúcio do pênis, decorrente da presença de fimose ou não, é um dos principais fatores relacionados com a progressão deste carcinoma, que promoverá uma alteração no tecido do pênis, causando tumor, feridas ou úlceras. Relacionado ao enfermeiro, são evidenciados que: a prática de educação em saúde, como por exemplo, orientar o paciente sobre os procedimentos corretos de higienização íntima, a realização do autoexame físico do genital, a avaliação das condições de higiene semanalmente e a conscientização sobre os riscos do Câncer Pênis, a importância do autoexame e o uso de preservativos são as formas direcionadas ao enfermeiro, para prevenir este tipo de câncer (Santos, 2018).

Quer dizer, por ser um dos poucos cânceres que podem ser evitados, é de fundamental importância de fazer o diagnóstico precoce desta patologia. Torna-se necessário, então, intensificar atividades como nas campanhas de prevenção, podendo, assim, incrementar estratégias como o autoexame, higiene intima adequada, a realização de postectomia e a prevenção de infecções sexualmente transmissíveis (Correia, 2018).

Significa que, o diagnóstico precoce contribui para a irradiação, controle, redução do crescimento local da doença o que leva a não amputação do órgão, a qual proporciona danos sexuais, psicológicos e físicos ao homem. Quando não alcançado a cura por outros métodos, o tratamento cirúrgico pode suscitar grades mudanças fazendo com que o homem reflita sobre seu próprio corpo, psique e âmbito social (Correia, 2018; Soares, 2019).

O enfermeiro dentro de suas competências realizada uma abordagem deliberada de resolução de problemas que caracteriza em processo de enfermagem, este possui uma visão 


\title{
RECIMA21 - REVISTA CIENTÍFICA MULTIDISCIPLINAR ISSN 2675-6218
}

\author{
ATUAÇÃO DO ENFERMEIRO FRENTE A PREVENÇÃO DO CÂNCER DE PÊNIS NA \\ ATENÇÃO PRIMÁRIA DE SAÚDE: UMA REVISÃO DA LITERATURA \\ Wanderson Alves Ribeiro, Joelma Zoroastro dos Santos Souza, Marluci Conceição dos Santos Almeida, \\ Lilian Laine da Conceição Dias, Renata Alves Fonseca, Vinícius Santos Braz da Cunha, \\ Greyce Kelly Souza Motta Alcoforado, Elisa Santana Daniel,
}

holística do paciente para atender as suas necessidades, incluindo avaliação, diagnostico de enfermagem, planejamento, implementação e analise final (Soares, 2019).

Diante desta realidade ressalta-se a importância da educação em saúde na prevenção de doenças relacionada a pratica sexual, o enfermeiro tem papel fundamental na sensibilização e na construção de novos saberes da comunidade. Essa contribuição do enfermeiro é essencial para o controle das Infecções Sexualmente Transmissíveis e na redução nas taxas de neoplasias penianas associadas ao papiloma vírus humano. Orientações a respeito do câncer de pênis e autoexame contribuem para diagnóstico precoce e aumentam as chances de bom prognostico (Passos, 2019).

Nessa forma, as contribuições deste estudo vão além do conhecimento, na medida em que pretende servir de subsídio para orientar ou definir ações no âmbito da educação em saúde, visando sensibilizar para adesão do autoexame e detecção precoce para o Câncer Pênis e, assim, contribuir significativamente para a redução da proporção de diagnósticos em estágios avançados desta doença que abala fisicamente e emocionalmente com os homens e sua família (Siqueira; Mantovani \& Kletemberg, 2019).

Certamente os diagnósticos de enfermagem encontrados nos pacientes submetidos a tratamento cirúrgicos para câncer de pênis estão relacionados com o procedimento cirúrgico e com as consequências físicas e psicológicas que ele acarreta. Este resultado pode ser justificado pelo fato de o câncer ter a cirurgia como forma de tratamento mais realizada. O enfermeiro no pós-operatório tem como atribuição avaliar e cuidar das feridas, evitando processo infeccioso e promovendo condições para uma boa cicatrização (Magalhães et al., 2015).

\section{CONSIDERAÇÕES FINAIS}

Conclui-se que este estudo possibilitou descrever e identificar os dados clínicos dos pacientes com câncer de pênis em nível primário, mas também secundário e terciário, que possuem semelhanças entre os autores citados, no sentido de enfatizar a educação em saúde por meio da divulgação de medidas preventivas.

Ao comparar as pesquisas selecionadas, conclui-se que os autores estão em consenso quanto a medidas de incentivo aos homens na busca de prevenção do câncer de pênis e quanto à necessidade de incremento do público masculino nas Unidades de Atenção Básica de Saúde. Particularmente, a figura masculina é vista como ser forte, invulnerável e resistente aos cuidados, fazendo com que eles evitem ir à busca de informações e cuidados nos locais onde é prestado serviços de saúde. 

ISSN 2675-6218

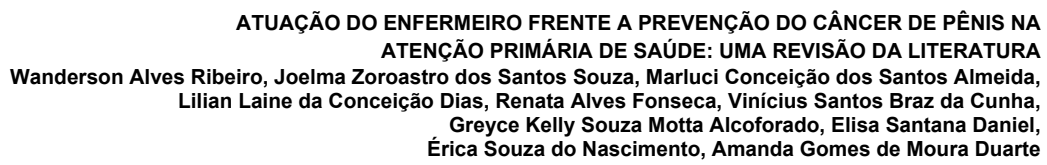

Por fim, ressalta-se a importância do desenvolvimento de pesquisas futuras por enfermeiros trazendo novas estratégias sobre a temática, bem como evidências para fortalecer os cuidados futuros de maneira holística aos pacientes.

\section{REFERÊNCIAS}

ANDRADE, M.; MARCONI, M.; LAKATOS, E. Fundamentos de metodologia científica: técnicas de pesquisa 7. ed. São Paulo: Atlas, 2010.

BARREIRA, M. A.; OLIVEIRA LIMA, L. et al. Experiência do Hospital Haroldo Juaçaba com Reconstrução Utilizando Retalhos Miocutâneos em Cirurgia para Tratamento do Câncer de Pênis locorregionalmente Avançado. Revista Brasileira de Cancerologia, v. 60, n. 1, p. 43-50, 2014.

BARROS, A. L. B. L. D., LOPES, J. D. L. A legislação e a sistematização da assistência de enfermagem. Enferm. foco (Brasília), p. 63-65, 2010.

BRASIL. Ministério da Saúde. Câncer de Pênis. Brasília: Instituto Nacional de Câncer (INCA), 2019.

BRASIL. Ministério da Saúde. Política Nacional de Atenção Integral à Saúde do Homem: princípios e diretrizes. Brasília: Secretaria de Atenção à Saúde, 2008.

CAMARGOS, B. F.; MATOS, L. R. P.; PENA, H. P. O paciente frente ao diagnóstico de câncer e a atuação dos profissionais de enfermagem: uma Revisão Integrativa de Literatura. Revista de Enfermagem do Centro-Oeste Mineiro, 2014.

COELHO, M. D. M. F.; MIRANDA, K. C. L. Educação para emancipação dos sujeitos: reflexões sobre a prática educativa de enfermeiros. Revista de Enfermagem do Centro-Oeste Mineiro, 2015.

CORREIA, A. S.; SILVA, G. V. F.; CHAGAS, H. M. et al. Câncer de pênis: Resultados de uma Campanha de Prevenção. Revista portal: saúde e sociedade, v. 3, n. 1, p. 628-638, 2018.

CONCEIÇÃO, V. M. D.; RAMOS, A. I.; ARAÚJO, J. S. et al. Determinantes sociais de pacientes com neoplasia peniana. Rev. enferm. UFPE on line, p. 338-345, 2019.

COSTA, L S.; TEIXEIRA, J. K. F.; CASTRO, S. F. Saberes e práticas do enfermeiro sobre câncer de pênis. Revista de Pesquisa: Cuidado é Fundamental Online, [s.I.], v. 7, n. 3, p. 2781, 2015.

DATA SUS. Números de amputações no Brasil. [s. l: s. e.], 2011.

DI PIETRO, M. L.; TELEMAN, A. A.; POSCIA, A. et al. Preventive newborn male circumcision: What is the child's best interest?. Cuadernos de bioética, v. 28, n. 3, p. 303-316, 2017.

GAO, W.; SONG, L. B.; YANG, J. et al. Risk factors and negative consequences of patient's delay for penile carcinoma. World journal of surgical oncology, v. 14, n. 1, p. 1-7, 2016.

Gil, A. C. Métodos e técnicas de pesquisa social. 6. ed. São Paulo: Atlas, 2008.

INCA. Um Problema da Saúde Pública. Brasília: INCA, 2013. 


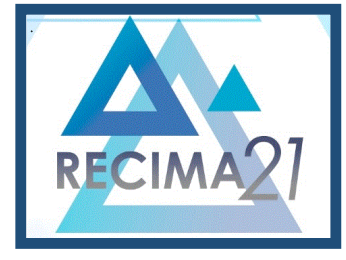

\title{
RECIMA21 - REVISTA CIENTÍFICA MULTIDISCIPLINAR ISSN 2675-6218
}

\author{
ATUAÇÃO DO ENFERMEIRO FRENTE A PREVENÇÃO DO CÂNCER DE PÊNIS NA \\ ATENÇÃO PRIMÁRIA DE SAÚDE: UMA REVISÃO DA LITERATURA \\ Wanderson Alves Ribeiro, Joelma Zoroastro dos Santos Souza, Marluci Conceição dos Santos Almeida, \\ Lilian Laine da Conceição Dias, Renata Alves Fonseca, Vinícius Santos Braz da Cunha, \\ Greyce Kelly Souza Motta Alcoforado, Elisa Santana Daniel, \\ Érica Souza do Nascimento, Amanda Gomes de Moura Duarte
}

JANINI, J. P.; BESSLER, D.; VARGAS, A. B. D. Educação em saúde e promoção da saúde: impacto na qualidade de vida do idoso. Saúde em Debate, v. 39, p. 480-490, 2015.

KLETEMBERG, D. F.; SIQUEIRA, M. D.; MANTOVANi, M. D. F. Uma história do processo de enfermagem nas publicações da Revista Brasileira de Enfermagem no período 1960-1986. Escola Anna Nery, v. 10, n. 3, p. 478-486, 2006.

MAGALHÃES, Â. M.; MATOS, A. É. B.; AGUIAR, M. I. F. Diagnósticos de enfermagem da CIPE® em pacientes submetidos a tratamento cirúrgico para câncer de pênis. Revista de Pesquisa Cuidado é Fundamental Online, v. 7, p. 163-172, 2015.

MINAYO, M. C. D. S. A produção de conhecimentos na interface entre as ciências sociais e humanas e a saúde coletiva. Saúde e Sociedade, v. 22, n. 1, p. 21-31, 2013.

NASCIMENTO, I. M.; MOREIRA, L. A.; RIBEIRO, W. A. A Saúde do Homem: Um estudo reflexivo na ótica das ações de promoção à saúde. Revista Pró-UniverSUS, v. 9, n. 2, p. 41-46, 2018.

NUNES, E. D. O desafio do conhecimento: pesquisa qualitativa em saúde. Ciência \& Saúde Coletiva, v. 12, n. 4, p. 1087-1088, 2007.

PAULA, S. H. B. D.; SOUZA, M. J. L.; ALMEIDA, J. D. Câncer de pênis, aspectos epidemiológicos e fatores de risco: tecendo considerações sobre a promoção e prevenção na Atenção Básica. BIS. Boletim do Instituto de Saúde (Impresso), v. 14, n. 1, p. 111-118, 2012.

PASSOS, J. F.; LIMA CZARNIAK, I.; CRUZ, J. P. M. Saúde do homem: o conhecimento dos caminhoneiros sobre o câncer de pênis. Revista Científica da Faculdade de Educação e Meio Ambiente, v. 10, n. 2, p. 107-119, 2019.

RIBEIRO, J. P.; CARDOSO, L. S.; PEREIRA, C. M. S. Assistência de enfermagem ao paciente oncológico hospitalizado: diagnósticos e intervenções relacionadas às necessidades psicossociais e psicoespirituais. Revista de Pesquisa: Cuidado é Fundamental Online, v. 8, n. 4, p. 51365142, 2016.

SANTOS, J. A. Autoexames mamário e testicular nos rastreamentos oncológicos: uma questão de prevenção quaternária?. Rev. bras. med. fam. comunidade, p. 1-6, 2015.

SANTOS, I. V.; BOMFIM, A. M. A.; LESSA, V. J. C. et al. Estratégias do enfermeiro na prevenção do câncer de pênis. Revista Uniabeu, v. 11, n. 29, p. 362-373, 2018.

SIQUEIRA, M. F. C.; SILVA ÁLVARES, M. B. et al. Conhecimento de homens universitários sobre câncer de pênis e práticas preventivas. Journal Health Npeps, v. 4, n. 1, p. 92-112, 2019.

SCHLENKER, B.; SCHNEEDE, P. The role of human papilloma virus in penile cancer prevention and new therapeutic agents. European urology focus, v. 5, n. 1, p. 42-45, 2019.

SOARES, D. F. D. S. Diagnóstico de enfermagem no pós-operatório de penectomia: revisão integrativa. Revista UNINGÁ, v. 56, n. S6, 2019.

SOUSA, J. E. R. B.; SOARES, L. S.; REIS, E. M. A. Conhecimento do homem sobre a prevenção de câncer de pênis. Revista de Enfermagem da UFPI, n. 3, v. 1, 2014. 